Elisa Maria de Aquino Lacerda'

Gilberto Kac"

Cynthia Braga da Cunha"I'

Maria do Carmo Leal"'
Departamento de Nutrição e Dietética. Instituto de Nutrição Josué de Castro (INJC). Universidade Federal do Rio de Janeiro (UFRJ). Rio de Janeiro, RJ, Brasil

" Departamento de Nutrição Social e Aplicada. INJC-UFRJ. Rio de Janeiro, RJ, Brasil

III Departamento de Epidemiologia e Métodos Quantitativos em Saúde. Escola Nacional de Saúde Pública Sérgio Arouca. Fundação Instituto Oswaldo Cruz. Rio de Janeiro, RJ, Brasil

Correspondência | Correspondence: Elisa Maria de Aquino Lacerda Instituto de Nutrição Josué de Castro Departamento de Nutrição e Dietética Centro de Ciências da Saúde - UFRJ Av. Carlos Chagas Filho, 373 Edifício do Centro de Ciências da Saúde Bloco J $-2^{\circ}$ andar, sala 26 Cidade Universitária

21941-590 Rio de Janeiro, RJ, Brasil

E-mail: elisalacerda@ufrj.br

\section{Consumo alimentar na gestação e no pós-parto segundo cor da pele no município do Rio de Janeiro}

\section{Food intake during pregnancy and postpartum according to skin color in Rio de Janeiro, Brazil}

\section{RESUMO}

OBJETIVO: Avaliar o consumo alimentar durante a gestação e pós-parto, segundo cor da pele.

MÉTODOS: Estudo longitudinal prospectivo que incluiu 467 mulheres entre 15 e 45 anos no período pós-parto, no município do Rio de Janeiro, entre 1999 e 2001. Foi aplicado um questionário de freqüência de consumo de alimentos aos 15 dias pós-parto (consumo referente ao período da gestação) e aos seis meses (consumo referente ao período pós-parto). Foi utilizada análise de covariância para analisar diferenças no consumo alimentar, segundo cor da pele, controlada pela escolaridade.

RESULTADOS: Durante a gestação, pretas e pardas apresentaram consumo de energia $13,4 \%$ e $9,1 \%(p=0,009$ e $p=0,028)$ e consumo de carboidrato $15,1 \%$ e $10,5 \%$ maior que brancas $(\mathrm{p}=0,005$ e $\mathrm{p}=0,014)$, respectivamente. Mulheres pretas e brancas apresentaram consumo energético $34 \%$ e $20 \%$ acima das recomendações nutricionais, respectivamente $(\mathrm{p}=0,035)$. Durante o período pós-parto, as pretas apresentaram consumo de energia 7,7\% maior e consumo de lipídios 14,8\% maior que as brancas; consumo de ácidos graxos saturados $23,8 \%$ maior que brancas $(p=0,003)$ e $13 \%$ maior que pardas $(p=0,046)$. A adequação de consumo de lipídios e ácidos graxos saturados foi maior em pretas que em brancas $(\mathrm{p}=0,024 \mathrm{e} \mathrm{p}=0,011$, respectivamente).

CONCLUSÕES: Os resultados mostram ser necessário revisar estratégias de intervenção nutricional no pré-natal e implementar assistência nutricional no pós-parto, para ajustar o consumo alimentar a níveis adequados, considerando as diferenças por cor/raça identificadas.

DESCRITORES: Consumo de alimentos. Gravidez. Lactação. Etnia e saúde. Estudos longitudinais. 


\section{ABSTRACT}

OBJECTIVE: To assess dietary intake during pregnancy and postpartum according to skin color.

METHODS: A longitudinal prospective study was carried out comprising 467 postpartum women aged between 15-45 years in the city of Rio de Janeiro, Southeastern Brazil, in 1999-2001. A food frequency questionnaire was administered at two weeks postpartum (intake covering the pregnancy period) and at six months postpartum (intake covering the postpartum period). Analysis of covariance was performed to evaluate differences in food intake among skin color groups, adjusted for educational level.

RESULTS: During pregnancy, black and mulatto women had $13.4 \%$ and 9.1\% higher energy intake $(\mathrm{p}=0.009$ and $\mathrm{p}=0.028)$ and $15.1 \%$ and $10.5 \%$ higher carbohydrate intake $(\mathrm{p}=0.005$ and $\mathrm{p}=0.014)$ than white women, respectively. Energy intake of black and white women exceeded the nutritional recommendations by $34 \%$ and $20 \%$, respectively $(\mathrm{p}=0.035)$. During the postpartum period, black women had $7.7 \%$ higher energy intake $(\mathrm{p}=0.030)$ and $14.8 \%$ higher lipid intake $(\mathrm{p}=0.008)$ than white women, as well as $23.8 \%$ and $13 \%$ higher saturated fatty acids intake than white $(\mathrm{p}=0.003)$ and mulatto $(\mathrm{p}$ $=0.046)$ women, respectively. The adequacy of lipid and saturated fatty acids intake was higher in black $(\mathrm{p}=0.024)$ than white women $(\mathrm{p}=0.011)$.

CONCLUSIONS: The study suggests the need to revise nutritional interventions strategies in the prenatal period, and to implement nutritional guidance programs during the postpartum period in order to adjust food intake to adequate levels, taking into consideration racial differences identified.

KEY WORDS: Food consumption. Pregnancy. Lactation. Ethnic group and health. Longitudinal studies.

\section{INTRODUÇÃO}

A nutrição materna exerce grande impacto no resultado da gestação e sobre a saúde da mulher e da criança durante a lactação. Em ambas as fases é fundamental que as recomendações nutricionais, que se encontram aumentadas em relação às mulheres adultas, sejam atendidas para garantir aporte nutricional e ganho de peso adequados. ${ }^{9}$

O consumo inadequado de vitaminas e minerais está associado a desfechos gestacionais desfavoráveis. Há elevada proporção de mulheres em idade reprodutiva que consome dietas com quantidades insuficientes de micronutrientes como zinco, ácido fólico, cálcio e ferro. ${ }^{1,9}$

O consumo energético é um importante determinante do ganho de peso durante a gestação que, por sua vez, é um dos fatores que sistematicamente vem apresentando associação positiva com a retenção de peso pósparto ${ }^{10-12} \mathrm{e}$ obesidade materna. ${ }^{8}$ Quantificar o consumo nutricional, principalmente o energético, durante a gestação e pós-parto é fundamental para contribuir para o esclarecimento de uma questão tão relevante, complexa e multifatorial como a obesidade.

Nos Estados Unidos, a obesidade tem apresentado prevalências mais elevadas na população negra, mesmo após ajuste para idade e educação., ${ }^{3,150}$ No Brasil, poucos estudos chegaram a resultados semelhantes, em virtude de as desigualdades étnico-raciais na ocorrência de obesidade terem sido pouco exploradas. ${ }^{4,5}$ Tendo em vista esta lacuna no conhecimento, explorar possíveis diferenças no consumo alimentar segundo cor da pele pode tornar-se um elemento que ajude a esclarecer a maior prevalência de excesso de peso e obesidade em mulheres negras. É plausível supor que as mulheres negras apresentem maior consumo energético que as brancas durante a gestação e o pós-parto.

O resultado da revisão de literatura* na base de dados

* A revisão de literatura utilizou as palavras-chave "food intake pregnancy", "food intake lactation" e "food intake postpartum", excluiu artigos de revisão, meta-análise, editorial e cartas, bem como artigos em idiomas diferentes do português: inglês, espanhol e francês, e não realizados com seres humanos. 
LILACS e MEDLINE, de 1993 a 2004, mostrou que somente quatro estudos avaliaram diferenças de consumo segundo cor da pele, tendo sido evidenciadas, em todos eles, diferenças entre o consumo de mulheres brancas e negras. ${ }^{2,7,18,22}$ Nenhum deles foi realizado no Brasil.

O objetivo do presente trabalho foi avaliar o consumo alimentar de mulheres na gestação e no pós-parto, segundo cor da pele.

\section{MÉTODOS}

Estudo longitudinal prospectivo incluindo mulheres no período pós-parto, usuárias de centro de saúde do município do Rio de Janeiro. A coleta de dados durou 24 meses, de maio de 1999 a abril de 2001. As mulheres foram entrevistadas em quatro ocasiões: com menos de 30 dias, aos dois, seis e aos nove meses após o parto. A captação ocorreu em três momentos e em três locais distintos: (a) no período pós-parto imediato, na principal maternidade da região ( $\mathrm{N}=229 ; 32,3 \%)$, (b) durante o terceiro trimestre do acompanhamento pré-natal, no serviço de saúde ( $\mathrm{N}=268 ; 37,8 \%)$ e (c) após o parto, quando as puérperas levavam seus filhos ao setor de puericultura do serviço de saúde para vacinação da BCG $(\mathrm{N}=212 ; 29,9 \%)$.

Os critérios de elegibilidade para a entrada na coorte foram: idade entre 15 e 45 anos, ausência de doenças crônicas, gestação de feto único, residência na área de abrangência do centro de saúde, idade gestacional no momento do parto igual ou superior a 37 semanas e intervalo de tempo entre o parto e a primeira entrevista inferior a 30 dias. ${ }^{10}$ As mulheres recrutadas, num total de 709, apresentaram similar perfil de idade, peso pré-gestacional, escolaridade e paridade..$^{10}$ Aceitaram participar do estudo 478 mulheres. Foram excluídas as mulheres que não responderam aos dois questionários de consumo alimentar $(\mathrm{N}=11)$, resultando em uma amostra final de 467 mulheres.

A avaliação do consumo alimentar foi realizada de forma retrospectiva. A primeira entrevista, realizada com menos de 30 dias pós-parto, incluiu questionário sobre consumo alimentar na gestação. A segunda entrevista ocorreu aos seis meses pós-parto e incluiu consumo alimentar durante o período pós-parto. Foi utilizado um questionário de freqüência de consumo alimentar, semiquantitativo, previamente validado, ${ }^{21}$ com 81 itens e oito opções de resposta. Para o cálculo da ingestão de energia e de nutrientes foi utilizada uma rotina desenvolvida em planilha eletrônica. As quantidades das porções eram multiplicadas pelo número de porções consumidas e, posteriormente, por uma freqüência específica para obter o consumo diário. Em seguida, esses valores diários obtidos eram transformados em energia e nutrientes de acordo com a Tabela Brasileira de Composição de Alimentos e, não sendo nela encontrado o alimento ou nutriente, a Tabela de Composição de Alimentos do Instituto Brasileiro de Geografia e Estatística (IBGE).

As variáveis principais do estudo foram: cor da pele (variável independente) e consumo alimentar (variável dependente). A cor da pele foi categorizada de acordo com a proposta do IBGE, sendo identificada pelo método da heteroatribuição de pertença de cor. O entrevistador recebeu treinamento prévio para classificação da cor da pele das mães com o objetivo de minimizar a ocorrência do viés de má-classificação.

As variáveis referentes ao consumo alimentar foram:

1) Consumo de energia, proteínas, carboidratos, lipídios, ácidos graxos saturados, colesterol, cálcio e ferro (variáveis contínuas).

2) Adequação de energia, proteínas, carboidratos, lipídios, ácidos graxos saturados, colesterol, cálcio e ferro (variáveis contínuas) - é a proporção do consumo de energia e nutrientes em relação às recomendações nutricionais preconizadas para a gestação e período pós-parto, pelas agências internacionais (Food and Agriculture Organization, National Institute of Health, Institute of Medicine). A adequação acima de 100\% para energia, lipídios, carboidratos, ácidos graxos saturados e colesterol foi interpretada como consumo excessivo e a adequação menor do que $100 \%$ para cálcio, ferro e proteína foi interpretada como consumo deficiente.

3) Contribuição percentual energética dos grupos de alimentos (variável contínua) - é a distribuição percentual de 14 grupos de alimentos no consumo energético diário (grupo de cereais, raízes e tubérculos, leguminosas, carnes leite, ovos, frutas, hortaliças, gorduras, açúcar, bebidas alcoólicas, condimentos, alimentos prontos e outros). Os grupos criados seguiram o mesmo tipo de agrupamento de alimentos utilizado na Pesquisa de Orçamentos Familiares 2002/2003.*

Foram estudadas covariáveis antropométricas, obstétricas e socioeconômicas para caracterizar o perfil da amostra. Em cada entrevista o peso da mulher era aferido em balança eletrônica digital (Filizola, capacidade $150 \mathrm{~kg}$ ) e a estatura era aferida em estadiômetro portátil (Holtain/Harpenden). As mulheres estavam sem sapatos e vestiam roupas leves sem acessórios. $\mathrm{O}$ peso pré-gestacional e o ganho de peso gestacional foram referidos pelas mulheres. A avaliação do estado nutricional pré-gestacional foi determinada pelo índice de massa corporal (IMC) pré-gestacional. A retenção de peso seis meses após o parto foi calculada como sendo a diferença entre o peso aos seis meses pós-parto (aferido) e o peso pré-gestacional. As variáveis obstétricas incluídas foram: número de gestações, paridade, realização de pré-natal ( $<\mathrm{e} \geq$ seis consultas). A escolaridade refe-

* Instituto Brasileiro de Geografia e Estatística. Pesquisa de orçamentos familiares, 2002-2003. Análise da disponibilidade domiciliar de alimentos e do estado nutricional no Brasil. Rio de Janeiro; 2004 
re-se ao número de anos de estudo da mulher. A renda familiar total mensal foi expressa em salário mínimo, vigente no momento da primeira entrevista.

Foi realizada análise comparativa de algumas covariáveis do estudo entre as mulheres que responderam ao primeiro e ao segundo questionário, utilizando os testes qui-quadrado e t-student. A análise de variância mostrou não haver diferenças significativas no consumo alimentar entre adolescentes e adultas, motivo pelo qual as adolescentes foram incluídas na análise. A avaliação da diferença entre as médias das variáveis relacionadas ao consumo alimentar e o cálculo das médias ajustadas, segundo cor da pele materna, foram realizados por meio da análise de covariância (ANCOVA), controlada pela covariável escolaridade. ${ }^{19}$ A escolaridade, um importante fator determinante do consumo alimentar, foi escolhida como um marcador de nível socioeconômico devido à sua estabilidade e menor propensão a distorções. ${ }^{6}$ Empregou-se o teste de múltiplas comparações de Sidak para testar diferenças entre pares de cor da pele. ${ }^{19}$ A premissa de homogeneidade dos coeficientes de regressão foi testada, bem como outras premissas também presentes no modelo de regressão linear, e nas variáveis fortemente assimétricas aplicou-se a trans- formação logarítmica. Em todas as análises adotou-se o nível de significância de 5\%. O programa estatístico utilizado foi o SPSS 10.0.1.

O projeto foi submetido e aprovado pela Comissão de Ética do Núcleo de Estudos de Saúde Coletiva da Universidade Federal do Rio de Janeiro. Todos os participantes assinaram termo de consentimento obtido de forma livre e espontânea após terem sido feitos todos os esclarecimentos necessários.

\section{RESULTADOS}

A amostra final foi constituída de 467 mulheres; 407 $(87,1 \%)$ e $308(66 \%)$ responderam ao questionário no primeiro e segundo momento, respectivamente. Não foram encontradas diferenças nas perdas de seguimento segundo idade, cor, escolaridade e IMC pré-gestacional. Por outro lado, mulheres com rendimento inferior ao primeiro e superior ao quarto quartil apresentaram maior perda de seguimento em relação às demais (Tabela 1). Não foram identificadas mulheres com cor da pele diferente de branca, parda ou preta. A média de idade para mulheres brancas, pardas e pretas foi, respectivamente, de 25,8, 24,1 e 25,5 anos

Tabela 1. Proporção de perdas de seguimento entre as entrevistas de 30 dias e seis meses após o parto, segundo variáveis de interesse em uma coorte de mulheres de 15 a 45 anos. Rio de Janeiro, RJ, 1999-2001.

\begin{tabular}{|c|c|c|c|c|}
\hline Variável & Observação inicial (N) & Perda de seguimento $(\mathrm{N})$ & Proporção de perdas (\%) & $p^{*}$ \\
\hline Idade (anos) & & & & 0,3771 \\
\hline $20-30$ & 328 & 85 & 25,9 & \\
\hline $30-40$ & 74 & 13 & 17,6 & \\
\hline $41-45$ & 6 & 2 & 33,3 & \\
\hline Cor da pele & & & & 0,3513 \\
\hline Branca & 153 & 38 & 24,8 & \\
\hline Parda & 177 & 38 & 21,5 & \\
\hline Preta & 77 & 23 & 29,8 & \\
\hline Renda familiar (SM) & & & & 0,0127 \\
\hline$\leq 2,0\left(1^{\circ}\right.$ quartil $)$ & 108 & 35 & 32,4 & \\
\hline $2,1-3,5$ ( $2^{\circ}$ quartil $)$ & 97 & 19 & 19,6 & \\
\hline $3,6-6,5$ ( $3^{\circ}$ quartil $)$ & 102 & 15 & 14,7 & \\
\hline$>6,5$ (4o quartil) & 90 & 25 & 27,8 & \\
\hline Escolaridade (anos) & & & & 0,5713 \\
\hline $0-4$ & 94 & 15 & 16,0 & \\
\hline$\geq 5$ & 265 & 36 & 13,6 & \\
\hline IMC pré-gestacional $\left(\mathrm{kg} / \mathrm{m}^{2}\right)$ & & & & 0,6959 \\
\hline$<19,8$ (baixo peso) & 91 & 23 & 25,3 & \\
\hline $19,8-26,0$ (normal) & 247 & 67 & 27,1 & \\
\hline $26,01-29,0$ (sobrepeso) & 45 & 13 & 28,9 & \\
\hline$>29,0$ (obesidade) & 24 & 4 & 16,7 & \\
\hline
\end{tabular}

* Valor de $\mathrm{p}$ referente ao teste de $\chi^{2}$ para proporções;

SM: Salário mínimo

IMC: Índice de Massa Corporal 


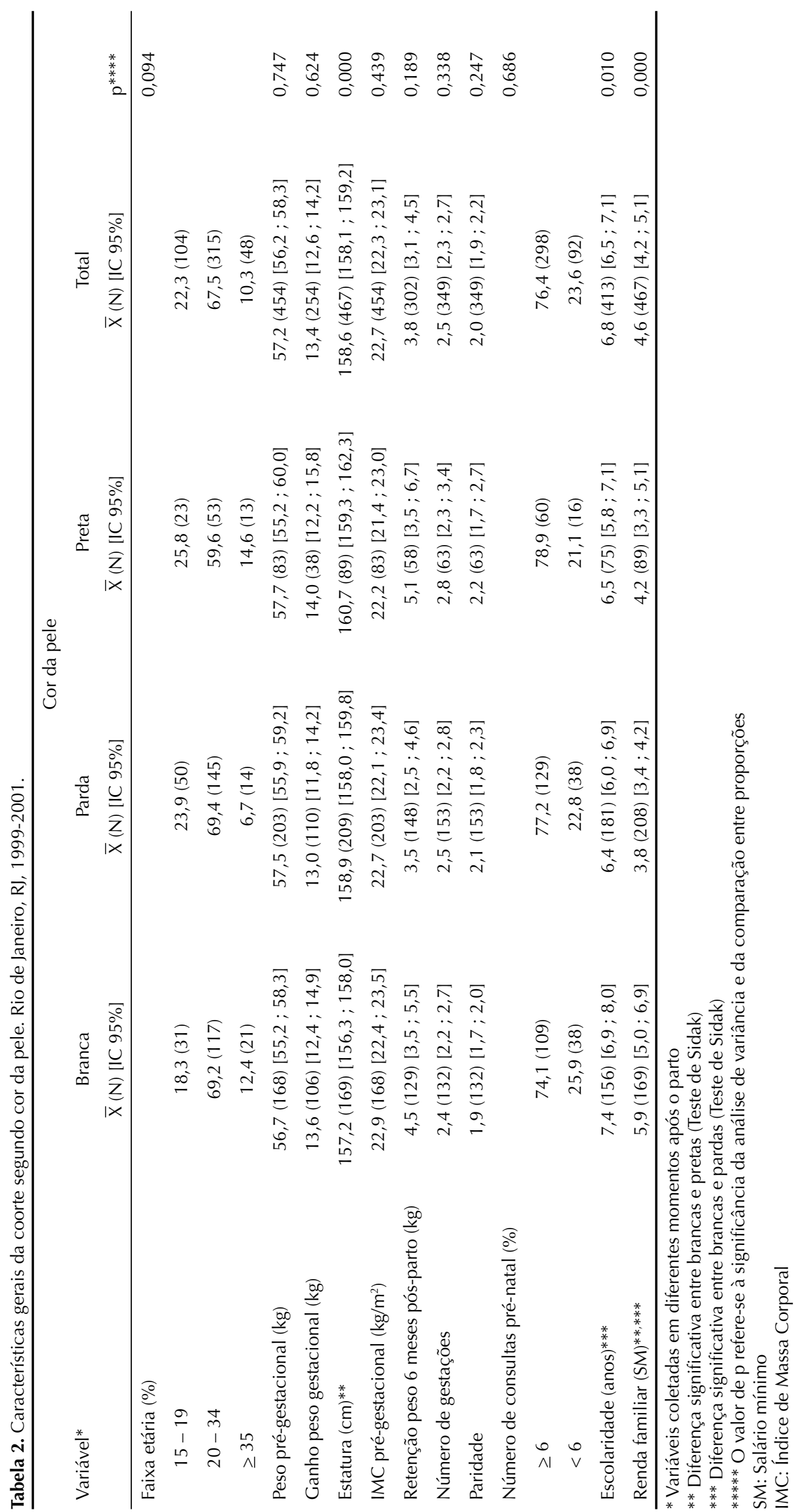


$(\mathrm{p}=0,040)$. Não foram observadas diferenças significativas quanto ao peso pré-gestacional, ganho de peso gestacional, IMC pré-gestacional, número de gestações, paridade e número de consultas de pré-natal segundo cor da pele. A estatura média de mulheres pretas foi maior do que a de mulheres brancas $(\mathrm{p}=0,000)$. Mulheres pardas apresentaram menor nível de escolaridade que brancas, e mulheres pardas e pretas apresentaram menor rendimento que brancas (Tabela 2).
A execução da ANCOVA preliminar para testar a hipótese da homogeneidade dos coeficientes de regressão das variáveis do consumo alimentar mostrou existir interação entre escolaridade e cor da pele para as seguintes variáveis: consumo de gorduras e de açúcar durante a gestação e consumo de cereal, carnes, frutas e bebidas alcoólicas durante o período pós-parto. Os modelos finais da ANCOVA e as médias ajustadas foram apresentados somente para as variáveis que não

Tabela 3. Consumo de nutrientes, adequação do consumo de nutrientes e contribuição energética de grupos de alimentos consumidos na gestação, segundo cor da pele, ajustado por escolaridade. Rio de Janeiro, RJ, 1999-2001.

\begin{tabular}{|c|c|c|c|c|}
\hline \multirow{3}{*}{ Variável } & \multicolumn{4}{|c|}{ Cor da pele } \\
\hline & Branca $(\mathrm{N}=155)$ & Parda $(\mathrm{N}=178)$ & Preta $(N=74)$ & Total $(\mathrm{N}=407)$ \\
\hline & $\bar{X}$ [IC 95\%] & $\bar{X}$ [IC 95\%] & $\bar{X}$ [IC 95\%] & $\bar{X}$ [IC 95\%] \\
\hline \multicolumn{5}{|l|}{ Consumo de nutrientes } \\
\hline Energia (kcal) & 2816 [2676;2957] & 3072 [2942;3203]* & $3192[2989 ; 3393]^{* *}$ & 3027 [2934;3119] \\
\hline Proteína (g) & $96[91 ; 100]$ & $102[98 ; 105]$ & $103[96 ; 110]$ & $100[97 ; 103]$ \\
\hline Carboidrato(g) & $457[433 ; 481]$ & $505[483 ; 528]^{*}$ & $526[491 ; 561] * *$ & $496[480 ; 512]$ \\
\hline Lipídios (g) & $67[63 ; 71]$ & $71[68 ; 75]$ & $75[70 ; 81]$ & $71[69 ; 74]$ \\
\hline AGS (g) & $29[27 ; 31]$ & $32[30 ; 33]$ & $32[30 ; 35]$ & $31[30 ; 32]$ \\
\hline Colesterol (mg) & $275[255 ; 295]$ & $298[279 ; 316]$ & $295[266 ; 324]$ & $289[276 ; 303]$ \\
\hline Cálcio (mg) & $817[763 ; 871]$ & $809[759 ; 860]$ & $832[755 ; 910]$ & $819[784 ; 855]$ \\
\hline Ferro $(\mathrm{mg}) * *$ & $14,6[13,8 ; 15,3]$ & $15,7[15,0 ; 16,4]$ & $16,3[15,2 ; 17,4] * *$ & $15,5[15,0 ; 16,0]$ \\
\hline \multicolumn{5}{|c|}{ Adequação percentual do consumo de nutrientes } \\
\hline Energia & $120[114 ; 126]$ & $129[124 ; 135]$ & $134[125 ; 143] * *$ & $128[124 ; 132]$ \\
\hline Proteína & $182[174 ; 191]$ & $191[183 ; 199]$ & $187[175 ; 200]$ & $187[181 ; 193]$ \\
\hline Carboidrato & $127[120 ; 135]$ & $140[133 ; 146]^{*}$ & $145[135 ; 155]^{* *}$ & $137[133 ; 142]$ \\
\hline Lipídios & $85[80 ; 91]$ & $90[85 ; 95]$ & $94[87 ; 102]$ & $90[87 ; 93]$ \\
\hline AGS & $123[116 ; 130]$ & $133[126 ; 139]$ & $134[123 ; 145]$ & $130[125 ; 135]$ \\
\hline Colesterol & $92[85 ; 98]$ & $100[93 ; 105]$ & $98[89 ; 108]$ & $96[92 ; 101]$ \\
\hline Cálcio & $82[76 ; 87]$ & $81[76 ; 86]$ & $83[75 ; 91]$ & $82[78 ; 86]$ \\
\hline Ferro & $54[51 ; 57]$ & $58[56 ; 61]$ & $60[56 ; 64] * *$ & $58[56 ; 59]$ \\
\hline \multicolumn{5}{|c|}{ Contribuição percentual energética dos grupos de alimentos } \\
\hline Cereal & $35,4[33,8 ; 37,1]$ & $38,6[37,0 ; 40,1]^{*}$ & $37,9[35,6 ; 40,2]$ & $37,3[36,2 ; 38,4]$ \\
\hline Tubérculo/raiz **** & $4,4[3,8 ; 5,0]$ & $4,2[3,7 ; 4,8]$ & $4,1[3,3 ; 5,0]$ & $4,2[3,9 ; 4,6]$ \\
\hline Leguminosas & $8,2[7,4 ; 9,0]$ & $8,7[7,9 ; 9,4]$ & $8,8[7,7 ; 10,0]$ & $8,6[8,0 ; 9,0]$ \\
\hline Carnes & $9,9[9,1 ; 10,7]$ & $9,6[8,9 ; 10,3]$ & $9,2[8,1 ; 10,3]$ & $9,6[9,0 ; 10,0]$ \\
\hline Leite & $8,1[7,4 ; 8,8]$ & $7,3[6,6 ; 8,0]$ & $6,5[5,5 ; 7,6] * *$ & $7,3[6,8 ; 7,8]$ \\
\hline Ovos**** & $0,7[0,6 ; 0,9]$ & $0,8[0,6 ; 0,9]$ & $0,6[0,4 ; 0,8]$ & $0,7[0,6 ; 0,8]$ \\
\hline Frutas & $11,8[10,7 ; 13,0]$ & $9,4[8,4 ; 10,4] *$ & $10,2[8,6 ; 11,8]$ & $10,5[9,8 ; 11,2]$ \\
\hline Hortaliças**** & $1,3[1,1 ; 1,5]$ & $1,1[0,9 ; 1,3]$ & $1,3[1,0 ; 1,6]$ & $1,2[1,1 ; 1,4]$ \\
\hline Gorduras**** & $* * *$ & $* * *$ & $* * *$ & $* * *$ \\
\hline Açúcar & $* * *$ & $* * *$ & $* * *$ & $* * *$ \\
\hline Bebidas alcoólicas ${ }^{* * * *}$ & $0,7[0,1 ; 1,3]$ & $0,9[0,4 ; 1,5]$ & $0,9[0,1 ; 1,8]$ & $0,8[0,4 ; 1,3]$ \\
\hline
\end{tabular}

* Diferença significativa entre brancas e pardas (Teste de Sidak)

** Diferença significativa entre brancas e pretas (Teste de Sidak)

*** ANCOVA não realizada pois resultados referentes a esses grupos de alimentos apresentaram interação entre escolaridade e cor da pele

**** Transformação logarítimica

AGS: Ácidos graxos saturados 
apresentaram interação entre cor da pele e escolaridade. Não foram incluídos os dados referentes aos grupos de alimentos denominados condimentos, alimentos prontos e outros alimentos uma vez que representaram juntos 3,5\% do consumo energético diário e não apresentaram diferenças segundo cor da pele.

Os dados referentes ao consumo alimentar durante a gestação constam da Tabela 3 . O consumo de energia proveniente de proteínas, lipídios, carboidratos e sa- carose representou, respectivamente, $13,5 \%, 21,1 \%$, $65,4 \%$ e $11,5 \%$ do consumo energético diário total, sem diferenças segundo cor da pele. Observou-se consumo excessivo de energia, proteína, carboidratos e ácidos graxos saturados (maior do que $100 \%$ ) e consumo deficiente de cálcio e ferro (menor do que 100\%). Mulheres pretas e pardas apresentaram maior consumo de energia ( $p=0,009$ e $p=0,028$, respectivamente) e de carboidratos $(p=0,005$ e $p=0,014$, respectivamente) que brancas.

Tabela 4. Consumo de nutrientes, adequação do consumo de nutrientes e contribuição energética de grupos de alimentos consumidos no pós-parto, segundo cor da pele, ajustado por escolaridade. Rio de Janeiro, RJ, 1999-2001.

\begin{tabular}{|c|c|c|c|c|}
\hline \multirow{3}{*}{ Variável } & \multicolumn{4}{|c|}{ Cor da pele } \\
\hline & Branca $(\mathrm{N}=114)$ & Parda $(\mathrm{N}=140)$ & Preta $(\mathrm{N}=54)$ & Total $(\mathrm{N}=308)$ \\
\hline & $\bar{x}$ [IC 95\%] & $\bar{x}$ [IC 95\%] & $\bar{X}$ [IC 95\%] & $\bar{x}$ [IC 95\%] \\
\hline \multicolumn{5}{|l|}{ Consumo de nutrientes } \\
\hline Energia (kcal) & 2016 [1876;2155] & 2172 [2046;2298] & $2339[2137 ; 2541]^{*}$ & $2175[2084 ; 2267]$ \\
\hline Proteína (g) & $71[67 ; 76]$ & $76[72 ; 80]$ & $80[73 ; 87]$ & $76[73 ; 79]$ \\
\hline Carboidrato(g) & $325[300 ; 350]$ & $353[331 ; 375]$ & $373[337 ; 408]$ & $350[334 ; 366]$ \\
\hline Lipídios (g) & $48[44 ; 52]$ & $51[47 ; 54]$ & $59[53 ; 64]^{*}$ & $52[50 ; 55]$ \\
\hline AGS (g) & $21[19 ; 23]$ & $23[21 ; 24]$ & $26[24 ; 29]^{*, * *}$ & $23[22 ; 24]$ \\
\hline Colesterol (mg) & $201[182 ; 219]$ & $212[196 ; 229]$ & $230[204 ; 256]$ & $214[202 ; 226]$ \\
\hline Cálcio (mg) & $522[474 ; 571]$ & $509[466 ; 552]$ & $518[448 ; 588]$ & $516[485 ; 548]$ \\
\hline Ferro $(\mathrm{mg})^{*}$ & $10,9[10,1 ; 11,7]$ & $11,7[11,0 ; 12,4]$ & $12,4[11,3 ; 13,6]$ & $11,7[11,2 ; 12,2]$ \\
\hline \multicolumn{5}{|c|}{ Adequação percentual do consumo de nutrientes } \\
\hline Energia & $101[93 ; 110]$ & $111[103 ; 118]$ & $118[106 ; 130]$ & $110[105 ; 116]$ \\
\hline Proteína & $107[99 ; 115]$ & $115[108 ; 122]$ & $117[106 ; 128]$ & $113[108 ; 118]$ \\
\hline Carboidrato & $117[106 ; 128]$ & $129[119 ; 138]$ & $136[120 ; 152]$ & $127[120 ; 135]$ \\
\hline Lipídios & $72[65 ; 79]$ & $78[72 ; 84]$ & $88[78 ; 98]^{*}$ & $79[75 ; 84]$ \\
\hline AGS & $105[95 ; 116]$ & $115[106 ; 124]$ & $132[117 ; 146]^{*}$ & $117[111 ; 124]$ \\
\hline Colesterol & $67[61 ; 73]$ & $71[65 ; 76]$ & $77[68 ; 85]$ & $71[67 ; 75]$ \\
\hline Cálcio & $52[47 ; 57]$ & $51[47 ; 55]$ & $52[45 ; 59]$ & $52[48 ; 55]$ \\
\hline Ferro & $121[113 ; 130]$ & $130[123 ; 138]$ & $138[126 ; 151]$ & $130[124 ; 136]$ \\
\hline \multicolumn{5}{|c|}{ Contribuição percentual energética dos grupos de alimentos } \\
\hline Cereal & $* * *$ & $* * *$ & $* * *$ & $* * *$ \\
\hline Tubérculo/raiz**** & $4,2[3,5 ; 4,8]$ & $3,7[3,0 ; 4,3]$ & $4,1[3,1 ; 5,1]$ & $4,0[3,5 ; 4,4]$ \\
\hline Leguminosas & $9,5[8,5 ; 10,5]$ & $10,3[9,4 ; 11,2]$ & $9,5[8,0 ; 10,9]$ & $9,8[9,1 ; 10,4]$ \\
\hline Carnes & $* * *$ & $* * *$ & $* * *$ & $* * *$ \\
\hline Leite & $6,3[5,4 ; 7,1]$ & $5,8[5,1 ; 6,5]$ & $5,4[4,2 ; 6,6]$ & $5,8[5,3 ; 6,3]$ \\
\hline Ovos**** & $0,7[0,6 ; 0,9]$ & $0,8[0,7 ; 1,0]$ & $0,7[0,5 ; 0,9]$ & $0,8[0,7 ; 0,9]$ \\
\hline Frutas & $* * *$ & $* * *$ & $* * *$ & $* * *$ \\
\hline Hortaliças**** & $1,4[1,1 ; 1,7]$ & $1,4[1,1 ; 1,6]$ & $1,7[1,3 ; 2,1]$ & $1,5[1,3 ; 1,7]$ \\
\hline Gorduras**** & $1,9[1,5 ; 2,2]$ & $2,0[1,7 ; 2,3]$ & $2,0[1,6 ; 2,5]$ & $2,0[1,7 ; 2,2]$ \\
\hline Açúcar & $13,7[12,3 ; 15,2]$ & $13,3[12,0 ; 14,6]$ & $13,7[11,5 ; 15,8]$ & $13,6[12,6 ; 14,5]$ \\
\hline Bebidas alcoólicas ${ }^{* * * *}$ & $* * *$ & $* * *$ & $* * *$ & $* * *$ \\
\hline
\end{tabular}

* Diferença significativa entre brancas e pretas (Teste de Sidak)

** Diferença significativa entre pardas e pretas (Teste de Sidak)

*** ANCOVA não realizada pois os resultados referentes a estes grupos de alimentos apresentaram interação entre escolaridade e cor da pele

**** Transformação logarítimica

AGS: Ácidos graxos saturados 
Mulheres pretas tiveram maior consumo de ferro do que brancas durante a gestação $(p=0,036)$, mas essa diferença desaparece se for considerado o consumo de ferro ajustado para energia $(5,1 \mathrm{mg} / 1000 \mathrm{kcal}$ em pretas e pardas e 5,2 mg/1000 kcal em brancas). Mulheres pretas e brancas apresentaram consumo energético $34 \%$ e $20 \%$ maior do que as recomendações nutricionais, respectivamente $(\mathrm{p}=0,035)$. O consumo de carboidratos em mulheres pretas e pardas foi $45 \%$ e $40 \%$ maior do que as recomendações, respectivamente, sendo significativamente maior do que o consumo observado em brancas ( $p=0,016 \mathrm{e} \mathrm{p}=0,041$, respectivamente). Observou-se que as mulheres pardas tiveram maior consumo energético proveniente de cereais $(p=0,021)$ e menor consumo energético proveniente de frutas do que brancas $(p=0,006)$, e as mulheres pretas, menor consumo energético proveniente do grupo de leite do que mulheres brancas $(\mathrm{p}=0,047)$.

A Tabela 4 mostra os dados referentes ao consumo alimentar no período pós-parto. O consumo de energia proveniente de proteínas, lipídios, carboidratos e sacarose representou $14,3 \%, 21,7 \%, 62,8 \%$ e $12,9 \%$, respectivamente, sem diferenças segundo cor da pele. Observou-se consumo excessivo de energia, proteína, carboidratos, ácidos graxos saturados e ferro (maior do que $100 \%$ ) e um consumo deficiente de cálcio (menor do que $100 \%$ ). Mulheres pretas apresentaram maior consumo de energia $(p=0,030)$ e de lipídios $(p=0,008)$ que brancas; e maior consumo de ácidos graxos saturados que brancas $(p=0,003)$ e pardas $(p=0,046)$. Mulheres pretas tiveram maior adequação de consumo de lipídios e ácidos graxos saturados que brancas ( $p=0,024$ $\mathrm{e} p=0,011$, respectivamente). Não foram encontradas diferenças significativas no consumo de grupos de alimentos, segundo cor da pele, no período pós-parto.

\section{DISCUSSÃO}

Indicadores de saúde que consideram cor ou raça/etnia são necessários para que se possa avaliar a qualidade de vida dos grupos populacionais raciais ou étnicos. Por meio deste recorte e após ajuste por escolaridade, constatou-se uma inadequação do consumo alimentar para todas as mulheres, com elevado consumo de energia durante a gestação em mulheres pretas e pardas; e elevado consumo de energia, lipídios e ácidos graxos saturados no pós-parto, em mulheres pretas. Todavia, o maior consumo energético observado em mulheres de cor preta e parda não se traduziu em uma dieta de melhor qualidade nutricional.

A principal limitação do presente estudo refere-se ao padrão classificatório de cor. Admite-se que a validade e confiabilidade da identificação de cor são limitadas, não sendo possível inferir que os indivíduos pertencem a esss categorias de maneira definitiva ou que cada categoria agrega um grupo absolutamente homogêneo. ${ }^{16}$ Alguns autores referem que o método de coleta de dados deve variar segundo o objetivo do estudo e que a heteroatribuição de pertença de cor permite estimar a exposição social a riscos à saúde. ${ }^{13,24}$

A determinação do consumo alimentar resulta da interação de múltiplos fatores, incluindo fatores socioeconômicos. O procedimento estatístico adotado na análise do efeito da cor da pele sobre o consumo alimentar permitiu verificar se o efeito da cor persiste após controlar para o efeito de uma variável socioeconômica, no caso a escolaridade. $O$ fato de mulheres de cor preta apresentarem estatura significativamente maior do que brancas e pardas é inesperado, mas não parece exercer influência sobre o consumo alimentar.

O presente estudo, realizado com mulheres de baixa renda e de área urbana, revelou uma série de inadequações nutricionais durante a gestação e pós-parto, com diferenças segundo cor da pele. $\mathrm{O}$ consumo energético excessivo na gestação, observado em maior intensidade em mulheres pretas e pardas, pode acarretar maior ganho de peso na gravidez e, conseqüentemente, maiores taxas de retenção de peso pós-parto. ${ }^{10,12} \mathrm{O}$ resultado observado em relação às pretas concorda com o estudo de Siega-Riz et al $^{22}$ que, utilizando questionário de freqüência de consumo de alimentos durante a gestação, mostrou que mulheres pretas consumiram dietas com maior teor energético que as brancas $(\mathrm{p}<0,01)$. Murphy $\&$ Abrams,${ }^{18}$ por outro lado, não encontraram diferenças significativas no consumo energético entre gestantes pretas e brancas.

Em relação ao período pós-parto, os dados encontrados no presente estudo se assemelham aos encontrados por Boardley et $\mathrm{al}^{2}$ em estudo com 345 mulheres, onde as pretas apresentaram significativamente maior consumo energético se comparadas com brancas, (2.039 versus $1.552 \mathrm{kcal}, \mathrm{p}=0,001)$. O consumo excessivo de carboidratos em mulheres pretas e pardas está coerente com o elevado consumo energético observado nessas mulheres. Siega-Riz et al $^{22}$ também encontraram maior consumo de carboidratos em gestantes pretas do em que brancas, apesar de as diferenças não terem sido significativas. Embora o consumo de sacarose não tenha diferido segundo cor da pele, o consumo médio desse nutriente ultrapassou o limite recomendado de $10 \%$ do consumo energético total. ${ }^{25}$

O consumo médio total de lipídios na gestação e pósparto foi menor do que o limite recomendado de $30 \%$ do valor energético da dieta $^{25}$ e menor do que o obtido por Siega-Riz et al. ${ }^{22}$ Esses autores observaram que gestantes pretas consumiam lipídios a $34,4 \%$ do valor energético da dieta, significativamente maior do que o encontrado em brancas. Boardley et $\mathrm{al}^{2}$ observaram que mulheres pretas e brancas apresentaram consumo de lipídios equivalente a $40,7 \%$ e $37,5 \%$ do consumo energético, respectivamente no pós-parto $(\mathrm{p}=0,001)$.

O maior consumo de ácidos graxos saturados, observado em pretas no pós-parto no presente estudo, é um 
importante fator de risco para doenças cardiovasculares. Isso pode ser justificado parcialmente pelo maior consumo de carne suína e embutidos (dados não apresentados) observado nessas mulheres. Além do consumo crescente de dietas com alta densidade energética e da atividade física reduzida, o consumo de dietas ricas em gorduras saturadas e açúcares destaca-se como uma das causas da obesidade. ${ }^{25}$

O consumo e adequação de cálcio durante a gestação e no pós-parto mostram uma dificuldade das mulheres em atenderem à recomendação desse mineral nessas importantes fases da vida. O consumo médio de cálcio na gestação foi menor do que o encontrado por Harville et $\mathrm{al}^{7}$ que observaram ingestão adequada de cálcio em mulheres pretas $(1.236 \mathrm{mg} / \mathrm{dia})$ e brancas $(1.258$ $\mathrm{mg} /$ dia). Comparando com o pós-parto, o maior consumo de cálcio na gestação pode refletir a tradição já bem disseminada de se aumentar o consumo de leite e derivados durante a gestação. Isso pode ser confirmado ao se observar, no presente estudo, a redução da contribuição percentual de consumo de alimentos do grupo do leite ocorrida no período pós-parto em relação à gestação. A Pesquisa de Orçamentos Familiares 2002-2003 $3^{10 *}$ destaca o fato de que a participação do grupo de leite e derivados na dieta tende a aumentar de forma uniforme com o nível de rendimento familiar. Entretanto, as pretas, apesar de não apresentaram a menor renda, foram as que apresentaram o menor consumo de leite na gestação.

A baixa adequação de ferro na gestação é um achado comum em países em desenvolvimento, decorrente do baixo consumo e/ou biodisponibilidade do ferro dietético e o elevado requerimento desse mineral. Durante o pós-parto, considerando que o consumo energético e de ferro foram menores que na gestação, a adequação de ferro encontrada reflete, provavelmente, o menor requerimento de ferro dessa fase da vida da mulher.

O consumo de bebidas alcoólicas durante a gestação está relacionado com importantes alterações físicas, cognitivas e comportamentais permanentes e irreversíveis do recém-nascido. Dessa forma, não há um nível seguro para consumo de bebidas alcoólicas e sua utilização é totalmente contra-indicada. ${ }^{9}$ No presente estudo, o consumo dessas bebidas durante a gestação foi baixo e não apresentou diferenças segundo cor da pele.

A desinformação sobre os cuidados de saúde na gestação e pós-parto poderia ocasionar negligência com o consumo alimentar desse período. Mulheres pretas são mais suscetíveis a adotar hábitos de vida insalubres, como resultado de discriminação e exclusão por elas sofrida ao longo da vida. ${ }^{14}$

Não se pode descartar a possível contribuição dos serviços de saúde à inadequação no consumo alimentar encontrada no presente estudo. Se não houve diferença no número de consultas de pré-natal segundo cor da pele, é possível supor que esteja ocorrendo iniqüidade na qualidade da oferta da atenção pré-natal. Essa iniqüidade é ocasionada pela desigualdade de tratamento aos grupos populacionais mais desfavorecidos socialmente, com distinção negativa para as mulheres de pele escura, como já referido por outros autores. ${ }^{14}$ Minorias étnicoraciais tendem a receber pior qualidade de assistência à saúde do que não-minorias, mesmo quando fatores relacionados ao acesso, como renda, são controlados. ${ }^{23}$ Essas disparidades são complexas e têm raízes em desigualdades históricas e contemporâneas, em vários níveis - sistema de saúde, processos administrativos, profissionais de saúde e pacientes. Em relação aos profissionais de saúde, as disparidades podem emergir da discriminação e, em relação ao paciente, de uma pobre interação com a equipe resultante de desconfiança e experiências anteriores insatisfatórias com os serviços de saúde. ${ }^{23}$

Embora o presente estudo não tenha sido desenhado para detectar tais questões, é fundamental verificar se as tendências aqui observadas se mantêm em outros estudos. Ainda assim, é necessário otimizar a relação com o paciente e, nesse sentido, deve-se por em prática o adequado acompanhamento do parto e puerpério, por meio da humanização da assistência obstétrica e neonatal. ${ }^{17,23}$ Os profissionais de saúde devem adotar uma postura ética e solidária, recebendo a mulher com dignidade, colocando em prática medidas e procedimentos benéficos para o acompanhamento pré-natal e pós-parto, inclusive aqueles referentes à atenção nutricional. ${ }^{23}$

Esforços devem ser realizados para que se intensifique o aconselhamento nutricional para todas as gestantes durante a assistência pré-natal, visando melhorar a qualidade da dieta durante a gestação. Adicionalmente, é necessário que se efetive um acompanhamento nutricional da mulher por um determinado período após o parto, a fim de reverter hábitos alimentares inadequados. Isso se aplica especialmente ao consumo de energia, lipídios e gordura saturada e atendimento às recomendações nutricionais específicas para essa fase da vida.

* Instituto Brasileiro de Geografia e Estatística. Pesquisa de orçamentos familiares, 2002-2003. Análise da disponibilidade domiciliar de alimentos e do estado nutricional no Brasil. Rio de Janeiro; 2004 


\section{REFERÊNCIAS}

1. Kaiser LL, Allen L, American Dietetic Association. Position of the American Dietetic Association: nutrition and lifestyle for a healthy pregnancy outcome. I Am Diet Assoc. 2002;102(10):1479-90.

2. Boardley DJ, Sargent RG, Coker AL, Hussey JR, Sharpe PA. The relationship between diet, activity, and other factors, and postpartum weight change by race. Obstet Gynecol. 1995;86(5):834-8.

3. Burke GL, Bild DE, Hilner JE, Folsom AR, Wagenknecht LE, Sidney S. Differences in weight gain in relation to race, gender, age and education in young adults: the CARDIA Study. Coronary Artery Risk Development in Young Adults. Ethn Health. 1996;1(4):327-35.

4. Chor D, Faerstein E, Kaplan GA, Lynch JW, Lopes CS. Association of weight change with ethnicity and life course socioeconomic position among Brazilian civil servants. Int J Epidemiol. 2004;33(1):100-6.

5. Franco LJ. Diabetes in Brazil - a review of recent survey data. Ethn Dis. 1992;2(2):158-65.

6. Galobardes B, Morabia A, Bernstein MS. Diet and socioeconomic position: does the use of different indicators matter? Int J Epidemiol. 2001;30(2):334-40.

7. Harville EW, Schramm M, Watt-Morse M, Chantala $\mathrm{K}$, Anderson J], Hertz-Picciotto I. Calcium intake during pregnancy among white and African-American pregnant women in the United States. I Am Coll Nutr. 2004;23(1):43-50.

8. Hill JO, Wyatt HR, Reed GW, Peters JC. Obesity and the environment: where do we go from here? Science. 2003;299(5608):853-5.

9. National Academy of Sciences. Institute of Medicine. Nutrition during pregnancy: Part I, Weight gain; Part II, Nutrient supplements. Washington; 1990.

10. Kac G, Benício MH, Velásquez-Meléndez G, Valente JG, Struchiner CJ. Gestational weight gain and prepregnancy weight influence postpartum weight retention in a cohort of Brazilian women. J Nutr. 2004;134(3):661-6.

11. Keppel KG, Taffel SM. Pregnancy-related weight gain and retention: implications of the 1990 Institute of Medicine guidelines. Am I Public Health. 1993;83(8):1100-3.

12. Lacerda EMA, Leal MC. Fatores associados com a retenção e o ganho de peso pós-parto: uma revisão sistemática. Rev Bras Epidemiol. 2004;7(2):187-200.
13. Laguardia J. O Uso da Variável "Raça" na pesquisa em saúde. Physis. 2004;14(2):197-234.

14. Leal MC, Gama SG, Cunha CB. Desigualdades raciais, sociodemográficas e na assistência ao pré-natal e ao parto, 1999-2001. Rev Saude Publica. 2005;39(1):1007

15. Lewis TT, Everson-Rose SA, Sternfeld B, Karavolos K, Wesley D, Powell LH. Race, education, and weight change in a biracial sample of women at midlife. Arch Intern Med. 2005;165(5):545-51.

16. Maio MC, Monteiro S, Chor D, Faerstein E, Lopes CS. Cor/raça no Estudo Pró-Saúde: resultados comparativos de dois métodos de autoclassificação no Rio de Janeiro, Brasil. Cad Saude Publica. 2005;21(1):171-180.

17. Programa de Humanização no Pré-natal e Nascimento Rev Bras Saude Mater Infant. 2002;2(1):69-71.

18. Murphy SP, Abrams BF. Changes in energy intakes during pregnancy and lactation in a national sample of US women. Am J Public Health. 1993;83(8):1161-3.

19. Neter J, Kutner MH, Nachtsheim CJ, Wasserman W. Applied Linear Statistical Model. 4 ed. Chicago: McGraw-Hill Education; 1996.

20. Sánchez-Johnsen LA, Fitzgibbon ML, Martinovich Z, Stolley MR, Dyer AR, Van Horn L. Ethnic Differences in Correlates of Obesity between Latin-American and Black Women. Obes Res. 2004;12(4):652-60.

21. Sichieri R. Estudo de Validação do Questionário de Freqüência de Consumo de Alimentos. In: Sichieri R, organizadora. Epidemiologia da Obesidade. 1 ed. Rio de Janeiro: Eduerj; 1998. p. 25-34.

22. Siega-Riz AM, Bodnar LM, Savitz DA. What are pregnant women eating? Nutrient and food group differences by race. Am J Obstet Gynecol. 2002;186(3):480-6.

23. Smedley BD, Stih AY, Nelson AR. Unequal treatment: confronting racial and ethnic disparities in health care. Washington: The National Academy Press; 2001.

24. Travassos C, Williams DR. The concept and measurement of race and their relationship to public health: a review focused on Brazil and the United States. Cad Saude Publica. 2004; 20(3):660-78.

25. World Health Organization, Food and Agriculture Organization. Diet, nutrition and the prevention of chronic diseases. Report of the joint WHO/FAO expert consultation. Geneva; 2003. (WHO Technical Report Series, 916). 